This provisional PDF corresponds to the article as it appeared upon acceptance. A copyedited and fully formatted version will be made available soon. The final version may contain major or minor changes.

\title{
A MULTIMODAL TRAINING WITH VISUAL BIOFEEDBACK IN SUBACUTE STROKE SURVIVORS: A RANDOMIZED CONTROLLED TRIAL
}

Emilia AMBROSINI, Elisabetta PERI, Claudia NAVA, Luca LONGONI, Marco MONTICONE, ALESSANDRA PEDROCCHI, Giorgio FERRIERO, SIMONA FERRANTE

European Journal of Physical and Rehabilitation Medicine 2019 Sep 26

DOI: 10.23736/S1973-9087.19.05847-7

Article type: Original Article

C 2019 EDIZIONI MINERVA MEDICA

Article first published online: September 26, 2019

Manuscript accepted: September 24, 2019

Manuscript revised: September 3, 2019

Manuscript received: May 10, 2019

Subscription: Information about subscribing to Minerva Medica journals is online at:

http://www.minervamedica.it/en/how-to-order-journals.php

Reprints and permissions: For information about reprints and permissions send an email to:

journals.dept@minervamedica.it - journals2.dept@minervamedica.it - journals6.dept@minervamedica.it 


\section{A MULTIMODAL TRAINING WITH VISUAL BIOFEEDBACK IN SUBACUTE STROKE SURVIVORS: A RANDOMIZED CONTROLLED TRIAL}

Emilia Ambrosini ${ }^{1 *}$, Elisabetta Peri ${ }^{2}$, Claudia $\mathrm{Nava}^{2}$, Luca Longoni ${ }^{2}$, Marco Monticone ${ }^{3,4}$, Alessandra Pedrocchi ${ }^{1}$, Giorgio Ferriero ${ }^{2}$, Simona Ferrante $^{1}$

${ }^{1}$ Neuroengineering and Medical Robotics Laboratory, Department of Electronics, Information and Bioengineering, Politecnico di Milano, Milan, Italy;

${ }^{2}$ Istituti Clinici Scientifici Maugeri IRCCS, Pavia, Italy;

${ }^{3}$ Department of Medical Sciences and Public Health, University of Cagliari, Cagliari, Italy;

${ }^{4}$ Department of Neuroscience and Rehabilitation, G. Brotzu Hospital, Cagliari, Italy.

\section{* Corresponding author:}

Emilia Ambrosini, Piazza Leonardo da Vinci, 32, 20133 Milan, Italy

phone: +390223999509; e-mail: emilia.ambrosini@polimi.it 


\begin{abstract}
BACKGROUND: Early interventions maximizing patient's involvement are essential to
\end{abstract} promote gait restoration and motor recovery after stroke.

AIM: To evaluate the effects of a multimodal biofeedback training involving cycling augmented by Functional Electrical Stimulation (FES) and balance exercises on walking ability and motor recovery.

DESIGN: Randomized controlled trial (NCT02439515).

SETTING: Inpatient rehabilitation facility.

POPULATION: Subacute stroke survivors (less than 6 months from the first event) with an age of up to 90 years old.

METHODS: 68 participants were randomly allocated to an experimental group, performing 15 sessions of biofeedback FES-cycling training followed by 15 sessions of biofeedback balance training (20 minutes each) in addition to usual care (70 minutes), and a control group performing 30 sessions (90 minutes) of usual care. Participants were evaluated before training, after 15 sessions, after 30 sessions, and at 6-month follow-up through: gait speed (primary outcome), spatio-temporal gait parameters, Six Minute Walking Test, Functional Independence Measure, Motricity Index, Trunk Control Test, Berg Balance Scale, and Fall Efficacy Scale.

RESULTS: Both groups significantly improved over time, but no group and interaction effects were found for any outcomes. The $73 \%$ of the experimental group achieved a clinically meaningful change in gait speed compared to the $38 \%$ of the control group ( $p$-value $=0.048$ ). These percentages were even more unbalanced for patients with a moderate to severe gait impairment at baseline (91\% versus $36 \%$; p-value $=0.008)$. 
CONCLUSIONS: The multimodal biofeedback training was not statistically superior to usual care, showing only a positive trend in favor of the experimental group on locomotion recovery. Patients initially not able to walk might be the best candidates for such a training.

CLINICAL REHABILITATION IMPACT: The multimodal biofeedback training is a taskspecific, repetitive and intensive training requiring a minimal supervision, which might result in a lower staff to patient ratio if organized in group sessions. Therefore, it can represent a good alternative for early stroke rehabilitation.

Keywords: Stroke; Rehabilitation; Randomized Controlled Trial; Biofeedback; Functional Electrical Stimulation. 


\section{INTRODUCTION}

Neurorehabilitation units are the primary services used in the subacute phase after stroke to promote functional recovery and regain independence. ${ }^{1}$ The prevalent old age of stroke survivors (about $50 \%$ aged $>75$ years and $30 \%>85$ years) ) $^{2,3}$ and the high prevalence of comorbidities (stroke occurs in isolation in $<6 \%$ of patients) ${ }^{4}$ negatively influences the success rate of rehabilitation,,$^{2,5}$ making the treatment and recovery for stroke increasingly complex. To maximize functional gains and shorten the length of hospital stays, it is generally recommended to start rehabilitation as soon as patients are clinically stable, no later than 30 days after stroke. ${ }^{6}$ Restoration of gait and gait-related activities is considered one of the primary goals of stroke rehabilitation. ${ }^{7}$ The guidelines for adult stroke rehabilitation include as key training parameters for improving mobility "activity-specific and functional task practice; practice that is progressively more difficult and challenging; practice that is of sufficient intensity, frequency, and duration; and practice that is at an appropriate time relative to stroke onset". ${ }^{1}$

Leg cycling training incorporates many of these elements: it is a functional task, sharing a similar locomotor pattern with walking, ${ }^{8,9}$ and can be performed with a sufficient intensity soon after stroke, since it does not require standing balance. ${ }^{10}$ Furthermore, the bike's rotating pedals may act as an external pacing cue, increasing the capability of even more severe patients to effectively pedal. ${ }^{11}$ Evidence shows that early cycling training significantly improves motor functions, balance ${ }^{12}$ and walking ability, ${ }^{13}$ therefore becoming an established intervention in subacute stroke rehabilitation. ${ }^{14}$

Leg cycling can be also combined with other therapies, such as Functional Electrical Stimulation (FES) and biofeedback, to improve its beneficial effects. ${ }^{10}$ FES-cycling training showed positive benefits on muscle strength, ${ }^{15,16}$ aerobic capacity, ${ }^{17}$ locomotion performance, ${ }^{18,19}$ and motor coordination. ${ }^{20}$ The use of visual biofeedback increases patient's attention during training, improves sensorimotor integration, and, consequently, improves 
training efficiency. ${ }^{1,21}$ Biofeedback cycling training led to improved leg functions, walking endurance and speed for patients with chronic stroke. ${ }^{22}$

Recovery of locomotion after stroke inevitably includes the recovery of balance. ${ }^{1}$ Typical balance deficits of people with hemiparesis are: wider postural sway; weight bearing asymmetry, with the center of pressure (COP) shifted towards the unaffected leg; and reduced limits of stability. ${ }^{23,24}$ The degree of postural asymmetry during stance well correlates with the level of gait impairment. ${ }^{25}$ Balance training augmented with visual force platform-based biofeedback is believed to be effective in improving balance control. A Cochrane review, including $7 \mathrm{RCTs}$, indicated that providing a feedback from a force platform resulted in improved stance symmetry of stroke survivors with a mean age of $<65$ years, but it did not improve clinical balance outcomes or measures of independence. ${ }^{26}$ More recently, the effects of biofeedback balance training were assessed on a group of 20 older stroke patients (age 6072 years) and significant improvements of static and dynamic balance were found. ${ }^{27}$

Although the promising results regarding the efficacy of biofeedback cycling training on chronic stroke survivors, ${ }^{22}$ randomized controlled trials (RCT) evaluating the effects in the subacute phase are still missing. Furthermore, the efficacy of the combination between biofeedback and FES during cycling was never evaluated. Also, for what concerns force platform-based balance training, results in the literature are still controversial. Above all, patients included in the majority of the cited studies have a mean age of $<65$ years, below the median age of stroke population in Europe (73 years). ${ }^{28}$

Based on these premises, our first hypothesis was that patients with subacute stroke would benefit from a multimodal biofeedback training consisting of a 3-week FES-augmented cycling training followed by a 3-week force platform-based balance training. Specifically, the multimodal biofeedback training, although it does not directly make the patient walk, might show better results than usual care on locomotion recovery. Indeed, it comprises a FES- 
augmented cycling training, which already showed positive effect on walking ability, ${ }^{18,19}$ and a training specifically focused on the recovery of standing balance control, an essential requirement for locomotion. ${ }^{25}$ Both training phases further benefitted from the use of a visual biofeedback to maximize patient's involvement. The order of the two training phases was chosen to follow the natural process of motor re-learning: first, participants underwent cycling training to recover muscle strength and motor control of the paretic leg without the need of upright balance control; then, participants were asked to stand up and to perform balance exercises to recover postural control during upright stance.

Our second hypothesis was that more impaired subjects, who are initially not able to walk, would benefit more from the biofeedback multimodal training. Indeed, cycling training performed in the first phase allowed a task-specific and intensive practice already in the first weeks after stroke with no risk for the patient.

In order to test these hypotheses, we conducted a RCT comparing the effects of the multimodal biofeedback training in addition to usual care to equally intensive usual care on walking abilities, independence in Activities of Daily Life (ADL), muscle force, trunk control, balance, and fear of falling. Subacute stroke survivors up to 90 years old were recruited to favor the generalizability of the results to the overall stroke population. 


\section{MATERIAL AND METHODS}

A single-blinded parallel-group RCT (allocation ratio 1:1), following the CONSORT recommendations, ${ }^{29}$ was conducted. The study was approved by the Hospital Ethical Committee (date of approval: 10/03/2014) and registered to clinicaltrial.gov (NCT02439515). Consecutive recruitment was conducted at the inpatient rehabilitation facility of Istituti Clinici Scientifici Maugeri (Lissone, Italy) from June 2015 to May 2018.

\section{Participants}

To be eligible, patients had to have a first stroke (either ischemic or hemorrhagic) less than 6 months before recruitment ${ }^{30}$ resulting in hemiparesis, an age of 18-90 years, lower limbs range of motion not precluding pedaling, reduced spasticity of leg muscles (Modified Ashworth scale $<2$ ), and the capability to keep sitting position for 30 minutes. Exclusion criteria were: major cognitive impairment preventing the capability to understand training instructions, cardiovascular or respiratory instability preventing physical training, other neurological diseases, spatial hemineglect, severe aphasia preventing communication with the therapist, major visual deficits, cardiac pacemakers, allergy to electrodes, or inability to tolerate FES.

Candidate patients were evaluated by a physiatrist, and those who satisfied the entry criteria were asked to give their written informed consent. Those who agreed were randomized to one of the treatment programs using a list of codes previously generated through a permuted-block randomization procedure. An automatic assignment system, developed in MATLAB, was used to conceal the allocation.

\section{Intervention}

Figure 1 schematizes the treatment programs. Both groups underwent 30 neuro-motor rehabilitative sessions, lasting 90 minutes each, 5 days/week. Participants of both groups were trained individually on a face-to-face basis by equally experienced physiotherapists. The rehabilitative session was tailored on the specific needs of each patient. 
The control group received usual care including stretching, muscular conditioning, exercises for trunk control, standing, cycling and walking, and upper limb rehabilitation. ${ }^{31}$

The experimental group underwent a biofeedback training consisting of 15 sessions of voluntary cycling augmented by FES followed by 15 sessions of platform-based balance training. Both trainings lasted 20 minutes and were supported by a visual feedback. The daily session was completed by 70 minutes of usual care, so as all participants were trained for the same amount of time. Physiotherapists received a manual about the experimental intervention and a 2-hour training particularly focused on FES-cycling was organized.

Cycling training was performed on a motorized cycle-ergometer (MOTOmed ${ }^{\circledR}$, Reck GmbH, see Figure 1) equipped with two force sensors mounted at the crank base (PowerForce ${ }^{\circledR}$, Radlabor GmbH, see Figure 1). ${ }^{32}$ During pedaling the mean forces produced at the right and left pedal were computed at the end of each revolution and were displayed to the subject by means of two vertical bars, as shown in Figure 1. The subject was asked to maintain a similar height of the two bars, while pedaling. When the two bars had a similar height, they were displayed in green, otherwise they were grey. ${ }^{33}$ The ergometer's motor kept a minimum speed of 20RPM. A current-controlled 8-channel stimulator (RehaStim ${ }^{\circledR}$, Hasomed GmbH) provided electrical stimuli through surface self-adhesive electrodes (Pals ${ }^{\circledR}$ electrodes, Axelgaard Manufacturing Co., Ltd.) applied on the quadriceps, hamstrings, gastrocnemius lateralis, and tibialis anterior of both legs. FES was synchronized to the cycling movement through a biomimetic stimulation strategy, reported in Figure $1 ;{ }^{18}$ the stimulation ranges were shifted by $180^{\circ}$ between the two legs. The pulse width and the stimulation frequency were fixed at $400 \mu \mathrm{s}$ and $20 \mathrm{~Hz}$, respectively, while the current amplitude was customized for each muscle and subject: values able to induce visible contractions, under pain tolerance threshold, were used for the affected leg, whereas values just above the sensory threshold were used for the 
unaffected leg. Current values were checked and eventually adapted daily to compensate for electrodes re-positioning and patient's status.

Balance training was performed during upright stance using a force platform (Balance board basic $^{\mathrm{TM}}$, NeuroCom ${ }^{\circledR}$ International, Inc., see Figure 1). Each session consisted of 6 exercises: \#1 maintain the COP in a central target for 1 minute; \#2 move the COP rhythmically in the medio-lateral and \#3 anterior-posterior direction, following a moving target displayed on the screen; \#4 move the COP sequentially from the central position towards 8 different targets placed around the center; \#5 move the COP following a target, whose movement simulates a step performed with the left and \#6 the right leg. All exercises, but the first one, lasted about 3 to 4 minutes. Exemplary visual feedback for the different exercises are displayed in Figure 1. Range of motion and pacing were customized for each subject and could be changed over training to keep the exercises challenging based on the personal judgement of the therapist. Subjects were allowed to sit and rest between exercises. A walker was placed in front of the subject to prevent falls, or to provide patient's support if needed.

To ensure that there was no variability in the administration of the treatment throughout the course of the study, a fidelity check based on a treatment manual was conducted both during each session and at the end of the program.

\section{Outcome measures}

The primary end-point was the between-group change of gait speed. Patients were asked to perform three times a 10-meter walking test at their preferred speed and the central part of each test was performed over a 6-meter sensorized mat (GAITRite ${ }^{\circledR}$, CIR Systems Inc.), in order to exclude the acceleration and deceleration phases from the analysis. The overall 18 meters performed over the GaitRite mat were used to compute the gait speed.

Secondary outcome measures were: the Six Minute Walking Test (6MWT), providing an estimate of walking endurance, ${ }^{34}$ the Functional Independence Measure, motor subscale (FIM- 
MS), evaluating the level of independence in performing ADLs; ${ }^{35}$ the Motricity Index, leg subscale (MI-Leg), evaluating the strength of the affected leg; ${ }^{36}$ the Trunk Control Test (TCT), assessing trunk control in supine and sitting positions; ${ }^{37}$ the Berg Balance Scale (BBS), evaluating standing balance ability; and the Fall Efficacy Scale, assessing the fear of falling. ${ }^{38}$ Additionally, gait spatio-temporal parameters, e.g. cadence, step length, step time, single and double support time, swing velocity of the affected leg, and a temporal symmetry index (ST ratio), computed as the ratio between the percentage stance time of the affected and unaffected leg, ${ }^{33}$ were derived from the GAITRite acquisitions, averaging all the steps performed across the three trials.

Participants were assessed before training (T1), after 15 sessions, i.e. at the end of the first intervention (T2), after the following 15 sessions, i.e. at the end of the second intervention (T3), and in a follow-up visit (T4) about 6 months after T3. Assessors, collecting the outcome measures, were unaware of group assignment. At T3, subjects were also asked to rate the Global Perceived Effect (GPE) of treatment on a 7-level Likert scale (1: help a lot; 7: make things worse). ${ }^{39}$

Using a specific form, patients were asked to report any serious and/or distressing symptoms they experienced during training. Patients were considered dropout by the therapists when $>3$ sessions were missed.

\section{Statistics}

Considering that the standard deviation of the gait speed for subacute stroke survivors was reported to be $22 \mathrm{~cm} / \mathrm{s}$ and that the minimal clinically important difference (MCID) was estimated as $16 \mathrm{~cm} / \mathrm{s},{ }^{40}$ a sample size of 58 patients was computed to achieve a power of $80 \%$ and a significance level of 0.05 . To allow a $15 \%$ drop-out rate, the sample size was set to 68 patients. 
T-test for independent samples for continuous outcome measures and Pearson Chi-squared test for frequencies were used to compare participants' characteristics at baseline between the two groups.

To deal with missing data, available-case methods were used. The Shapiro-Wilk test was used to verify if the outcome measures were normally distributed. If the hypothesis of normality was not rejected, linear mixed model analyses for repeated measures ( $p$-value $<0.05)$ were carried out on each outcome measure, with group and time entered as fixed effects, time by group as interaction term, and the outcome as dependent variable. Otherwise, if the hypothesis of normality was rejected, the generalized linear model, which does not require the data to be normally distributed, was used.

To further investigate the effect of the intervention on locomotion, a dichotomic analysis was performed for the gait speed derived from the GaitRite mat and the distance covered during the 6MWT. For this analysis, only subjects who completed the T3 assessment were included. Participants of each intervention group were divided into two categories: improved, i.e. gait speed or $6 \mathrm{MWT}$ change from baseline $>\mathrm{MCID}\left(\mathrm{MCID}=16 \mathrm{~cm} / \mathrm{s}\right.$ for gait speed $;{ }^{40} \mathrm{MCID}=71 \mathrm{~m}$ for $6 \mathrm{MWT}^{41}$ ), versus unchanged/deteriorated. The proportions of improved subjects were compared between the two intervention groups by means of the Pearson Chi-squared test. The dichotomic analysis was repeated for the sub-group of older subjects (age $\geq 75$ years) and for the sub-group of more impaired subjects, identified as patients who were unable to walk or registered a gait speed $<40 \mathrm{~cm} / \mathrm{s}^{42}$ at baseline.

Finally, the between-group difference in terms of GPE scores was analyzed using the MannWhitney U test. The statistical analysis was performed using SPSS v25. 


\section{RESULTS}

A total of 426 subjects with a diagnosis of stroke were screened and 68 were recruited, randomized and included in the analysis, as shown in Figure 2. The most frequent reasons for exclusion were: second stroke (29\%), unable to understand training instructions $(19 \%)$, cardiovascular instability (12\%), bilateral motor impairment (9\%). The drop-out rates were 7\% at $\mathrm{T} 2,24 \%$ at $\mathrm{T} 3$, and $53 \%$ at $\mathrm{T} 4$.

Groups were comparable at baseline (Table I): participants had a mean age of 75 years and were recruited about two weeks after stroke. A sub-group of 15 subjects were not able to walk at baseline: they were slightly unbalanced between the two intervention groups ( 5 in the experimental group and 10 in the control group), but the two proportions were not significantly different ( $p$-value $=0.144)$.

The hypothesis of normality was rejected for MI and TCT scores; therefore, for these outcome measures, the generalized linear model was used. Table II summarizes the scores of the gait speed (primary outcome) and of the clinical outcome measures over time and between groups: all of the outcomes showed a significant time effect indicating an overall improvement of motor performance, while interaction effect was never highlighted. A significant group effect was found only for TCT, due to better scores achieved by the experimental group already at baseline. Although not significant, it can be noticed a trend in favor of the experimental group; indeed, the T3-T1 change was larger for the experimental group for all outcomes but TCT and Fall Efficacy Scale. The improvements were mostly maintained in both groups at follow-up. However, results at follow-up have to be considered with caution due to the high drop-out rate. Table III reports the scores on spatio-temporal gait parameters. A significant effect of time was found for all outcomes but ST ratio, while no group and interaction effects were highlighted. Also for what concerns spatio-temporal gait parameters, a trend, although not significant, in 
favor of the experimental group could be highlighted; indeed, the T3-T1 change of all outcome measures was larger for the experimental group.

Figure 3 summarizes the results of the dichotomic analysis on walking ability. At T3, a significant association between intervention and gait speed improvement ( $p$-value $=0.048$ ) was found, with $73 \%$ of the experimental group and only $46 \%$ of the control group improving the gait speed of a clinically important extent (Figure 3-A). When considering older patients, similar proportions were found with $67 \%$ of the experimental group who clinically improved the gait speed compared to the $38 \%$ of the control group (Figure 3-B); however, probably due to the lower sample size $(\mathrm{N}=31)$, the proportions did not differ significantly $(\mathrm{p}=0.104)$. Finally, when the sub-group of the most impaired subjects was considered (Figure 3-C), the proportion of patients who reported a meaningful locomotor recovery was significantly higher $(91 \%)$ in the experimental group compared to controls $(36 \%)$ (p-value $=0.008)$. For what concerns walking endurance derived from the 6MWT, the proportion of improved subjects in the experimental group (77\%) was significantly higher than the one of the control group (46\%), with a p-value of 0.023 (Figure 3-D). When only older or more impaired subjects were considered, the percentage of improved subjects was still higher in the experimental group, but no significant differences were found.

All patients perceived the intervention as very useful: the median (interquartile range) of GPE was 1 (1) for both groups (p-value=0.940).

The compliance to the intervention programs was very high since all patients missed less than 3 sessions. Neither of the interventions cause any serious adverse event. 


\section{DISCUSSION}

This RCT showed that a biofeedback FES-cycling and balance training in addition to usual care was not statistically superior to usual care of equal intensity in improving walking ability, daily life independence, muscle force, trunk control and balance in subacute stroke survivors. Indeed, both groups significantly improved the gait speed and the distance walked in six minutes of a clinically significant extent $\left(>16 \mathrm{~cm} / \mathrm{s}\right.$ for gait speed $^{40}$ and $>71 \mathrm{~m}^{41}$ for $6 \mathrm{MWT}$, Table II). Also, all the other clinical outcome measures, as well as the spatio-temporal gait parameters, significantly improved in both groups. Based on these results, we had to refuse our first hypothesis that a multimodal biofeedback training could outperform usual care in improving walking ability and motor recovery.

However, a non-significant trend of higher improvement in favor of the experimental group could be highlighted: the gait speed improved of about $42 \%$ and $37 \%$ in the experimental and in the control group, respectively. The relative improvements at the end of the intervention for what concerns walking endurance were $39 \%$ and $30 \%$ for the experimental and the control group, respectively (Table II). This positive trend was confirmed by the dichotomic analysis on locomotion performance (Figure 3). Indeed, the proportion of individuals who reported a clinically meaningful change in gait speed and walking endurance after treatment was significantly higher in the experimental group (about 75\%) than in the control group (less than $50 \%$, Figure 3-A/D). The results of the dichotomic analysis are stronger than the ones of the linear mixed model analysis, and this can be explained by the different way in which missing data were considered: in the linear mixed model analysis, if a subject was not able to perform the walking test, it was considered as a missing data. In the dichotomic analysis, instead, if a patient, initially not able to walk, achieved a gait speed >MCID at T3, it was considered as clinically improved. In the experimental group, 5 patients were not able to walk at baseline (Table I); at post-training, two of them were drop-outs, and therefore excluded from the 
analysis, and 3 were considered as clinically improved. Conversely, in the control group, 10 patients were not able to walk at T1 (Table I: at T3, 2 were drop-outs, 6 did not improve their gait speed of a clinically important extent ( 5 of them were still unable to walk), and only 2 were considered clinically improved. This unbalance between the two groups might explain the difference in the results of the two analyses.

The novel training proposed to the experimental group is shaped to meet the guidelines for adult stroke rehabilitation. ${ }^{1}$ First, both cycling and balance training maximize patient's involvement, taking advantage of visual biofeedback, and motor relearning as they are based either on cyclic (cycling) or on repetitive (balance) movements. ${ }^{43}$ Further, cycling training combines FES with the residual voluntary effort to enhance FES therapeutic effects. ${ }^{44-46}$ The two phases of the intervention were applied in sequence, favoring gradual skill acquisition. The characteristics of the multimodal biofeedback training made it safe, applicable soon after stroke, and cost-effective since it does not require a continuous one-to-one supervision of the therapist and may reduce the time spent by the therapist with individual patient. Organizing the inpatient rehabilitation in a supervised group setting - at least during the first weeks since admission when patients are not yet able to walk - the multimodal biofeedback training may increase therapy time without increasing staff time. ${ }^{47}$ FES-cycling and platform balance training could be part of a task-oriented circuit class training in which patient with subacute stroke practice tasks repetitively with ongoing progression under supervision of a therapist. The majority of the participants (78\%) were already able to walk at recruitment and this might partially explain why a statistical superiority of the biofeedback training with respect to usual care was not found. Indeed, the biofeedback training was designed to involve patients, initially not able to walk, in an intensive and task-oriented practice finalized to the recovery of locomotion, as soon as possible after the acute event. However, we did not restrict the 
recruitment only to subjects not able to walk, since this would have prevented to reach the desired sample size in a reasonable amount of time.

When considering more impaired patients (not able to walk or with a gait speed $<40 \mathrm{~cm} / \mathrm{s}$ ), the association between gait recovery and intervention was further supported. Indeed, after training, the clinical meaningful gait speed change was reported by more than $90 \%$ of the experimental group and less than $40 \%$ of the control group (Figure 3-C). This result seemed to support our second hypothesis that the biofeedback training is particularly recommendable to patients with a more severe walking impairment, who could not benefit in an early phase from a standard gait training, ${ }^{31}$ and it is in line with the results of previous studies showing that technology-assisted walking training is more effective in more severe patients. ${ }^{48,49}$ However, the small sample size of severe patients does not allow us to drive final conclusions.

The association between treatment and locomotion improvement showed a trend in favor of the experimental group also when restricting the sample to older participants (Figure 3-B/E). This result confirms the importance of devoting efforts in the rehabilitation of older stroke patients who can still benefit from an intensive training program. ${ }^{50}$ Older patients can benefit from the novel treatment despite it is based on innovative technologies and it does not require a one-to-one continuous interaction between patient and therapist, two aspects not frequently suggested to elderlies.

The improvements achieved at the end of the intervention seemed to be maintained for at least six months. However, the very high dropout rate at T4 (53\%) does not allow us to drive any kind of conclusion about the medium-term effect of the multimodal biofeedback training. A well-recognized limitation of the majority of RCTs on stroke patients is that the mean age of the participants is significantly lower than the average age of stroke population. ${ }^{4}$ Conversely, in this RCT the mean age of the participants perfectly matches the average age of stroke population $(73 \text { years })^{28}$ and this might favor the generalizability of the results. 
This study has some limitations. First, the dropout rates were quite high $(24 \%$ at $\mathrm{T} 3$ and $53 \%$ at T4) so as to decrease the statistical power of our results. The dropout rate at T3 was probably due to the participants old age (10 out of 16 people who dropped out from the study before the end of the intervention were $>75$ years old) and to the length of the intervention ( 6 weeks, 30 sessions), which is very close to the average stay in rehabilitation facilities after stroke in Italy ( 8 weeks). Similarly, the dropout rate at T4 was mainly due to the participants old age that prevented them to come back to the rehabilitation facility 6 months after discharge. Second, the enrolment criteria were quite strict, as highlighted by the small number of recruited subjects (68) compared to the whole primary cohort (426). The strictest criterium was the choice to recruit only patients experiencing a first stroke, which has limited the potential number of subjects who could benefit from the intervention, negatively impacting the external validity of the study. Thirdly, the presence of post-stroke depression was not analyzed, although this is an important factor that may predict positive or negative results of post-stroke rehabilitation interventions. Fourthly, the training effects on brain plasticity were not evaluated and therefore we could not elucidate the central mechanisms based on which motor recovery occurred. Lastly, patients were recruited in the subacute phase (about two weeks after stroke) and therefore the spontaneous recovery might have covered the training-specific effects.

\section{CONCLUSIONS}

In conclusion, this RCT did not support the hypothesis that a multimodal biofeedback training including FES-cycling and balance training in addition to usual care is superior to usual care of equal intensity in promoting walking, functional recovery, and balance ability in subacute stroke survivors. However, a trend in favor of the biofeedback training, mainly for what concerns walking ability, was found. These results, as well as the possibility to train more than one patient simultaneously if organized in group sessions, sustain the application of the 
biofeedback cycling and balance training in early stroke rehabilitation. Subjects with severe walking impairment seem to benefit more from this intervention. Future research should further investigate the effects of the biofeedback training on patients who are initially not able to walk and should evaluate the cost-benefit aspects of the novel intervention. The possibility to prolong the intervention at home after discharge should also be analyzed. 


\section{REFERENCES}

1. Winstein CJ, Stein J, Arena R, et al. Guidelines for Adult Stroke Rehabilitation and Recovery: A Guideline for Healthcare Professionals from the American Heart Association/American Stroke Association. Stroke 2016; 47: e98-e169.

2. Lui SK, Nguyen MH. Elderly Stroke Rehabilitation: Overcoming the Complications and Its Associated Challenges. Curr Gerontol Geriatr Res 2018; 2018: 1-9.

3. Benjamin EJ, Blaha MJ, Chiuve SE, et al. Heart Disease and Stroke Statistics - 2017 Update: A Report From the American Heart Association. Circulation; 135.

4. Nelson MLA, Mckellar KA, Yi J, et al. Stroke rehabilitation evidence and comorbidity: a systematic scoping review of randomized controlled trials. Top Stroke Rehabil 2017; 24: 374-380.

5. Karatepe AG, Gunaydin R, Kaya T, et al. Comorbidity in patients after stroke: Impact on functional outcome. J Rehabil Med 2008; 40: 831-835.

6. Salter K, Jutai J, Hartley M, et al. Impact of early vs delayed admission to rehabilitation on functional outcomes in persons with stroke. J Rehabil Med 2006; 38: $113-117$.

7. Bergmann J, Krewer C, Bauer P, et al. Virtual reality to augment robot-assisted gait training in non-ambulatory patients with a subacute stroke: a pilot randomized controlled trial. Eur J Phys Rehabil Med 2018; 54: 397-407.

8. Raasch CC, Zajac FE. Locomotor Strategy for Pedaling: Muscle Groups and Biomechanical Functions. J Neurophysiol 1999; 82: 515-525.

9. Mazzocchio R, Meunier S, Ferrante S, et al. Cycling, a tool for locomotor recovery after motor lesions? NeuroRehabilitation 2008; 23: 67-80.

10. Barbosa D, Santos CP, Martins M. The application of cycling and cycling combined with feedback in the rehabilitation of stroke patients: A review. J Stroke Cerebrovasc 
Dis 2015; 24: 253-273.

11. Snijders AH, Bloem BR. Cycling for Freezing of Gait. N Engl J Med 2010; 362: e46.

12. Katz-Leurer M; Sender I; Keren O; Dvir Z. The influence of early cycling training on balance in stroke patients at the subacute stage. Results of a preliminary trial. Clin Rehabil 2006; 20: 398-405.

13. Tang A, Sibley KM, Thomas SG, et al. Effects of an Aerobic Exercise Program on Aerobic Capacity, Spatiotemporal Gait Parameters, and Functional Capacity in Subacute Stroke. Neurorehabil Neural Repair 2009; 23: 398-406.

14. Vanroy C, Feys H, Swinnen A, et al. Effectiveness of Active Cycling in Subacute Stroke Rehabilitation: A Randomized Controlled Trial. Arch Phys Med Rehabil 2017; 98: 1576-1585.e5.

15. Ferrante S, Pedrocchi A, Ferrigno G, et al. Cycling induced by functional electrical stimulation improves the muscular strength and the motor control of individuals with post-acute stroke. Europa Medicophysica-SIMFER 2007 Award Winner. Eur J Phys Rehabil Med 2008; 44: 159-67.

16. de Sousa DG, Harvey LA, Dorsch S, et al. Functional electrical stimulation cycling does not improve mobility in people with acquired brain injury and its effects on strength are unclear: a randomised trial. J Physiother 2016; 62: 203-208.

17. Lee SY, Kang SY, Im SH, et al. The effects of assisted ergometer training with a functional electrical stimulation on exercise capacity and functional ability in subacute stroke patients. Ann Rehabil Med 2013; 37: 619-627.

18. Ambrosini E, Ferrante S, Pedrocchi A, et al. Cycling Induced by Electrical Stimulation Improves Motor Recovery in Postacute Hemiparetic Patients. Stroke 2011; 42: 1068 1073.

19. Bauer P, Krewer C, Golaszewski S, et al. Functional electrical stimulation-assisted 
active cycling - Therapeutic effects in patients with hemiparesis from 7 days to 6 months after stroke: A randomized controlled pilot study. Arch Phys Med Rehabil 2015; 96: 188-196.

20. Ambrosini E, Ferrante S, Ferrigno G, et al. Cycling induced by electrical stimulation improves muscle activation and symmetry during pedaling in hemiparetic patients. IEEE Trans Neural Syst Rehabil Eng 2012; 20: 320-330.

21. Huang H, Wolf SL, He J. Recent developments in biofeedback for neuromotor rehabilitation. J Neuroeng Rehabil 2006; 3: 11.

22. Yang HC, Lee CL, Lin R, et al. Effect of biofeedback cycling training on functional recovery and walking ability of lower extremity in patients with stroke. Kaohsiung $J$ Med Sci 2014; 30: 35-42.

23. Nichols DS. Balance retraining after stroke using force platform biofeedback. Phys Ther 1997; 77: 553-8.

24. Geiger RA, Allen JB, O'Keefe J, et al. Balance and mobility following stroke: effects of physical therapy interventions with and without biofeedback/forceplate training. Phys Ther 2001; 81: 995-1005.

25. Nardone A, Godi M, Grasso M, et al. Stabilometry is a predictor of gait performance in chronic hemiparetic stroke patients. Gait Posture 2009; 30: 5-10.

26. Barclay-Goddard RE, Stevenson TJ, Poluha W, et al. Force platform feedback for standing balance training after stroke. Cochrane Database Syst Rev 2004; CD004129.

27. Maciaszek J. Effects of Posturographic Platform Biofeedback Training on the Static and Dynamic Balance of Older Stroke Patients. J Stroke Cerebrovasc Dis 2018; 27: 1969-1974.

28. The European Registers of Stroke (EROS) Investigators. Incidence of Stroke in Europe at the Beginning of the 21st Century. Stroke 2009; 40: 1557-1563. 
29. Altman DG, Moher D, Schulz KF. Improving the reporting of randomised trials: the CONSORT Statement and beyond. Stat Med 2012; 31: 2985-97.

30. Bernhardt J, Hayward KS, Kwakkel G, et al. Agreed Definitions and a Shared Vision for New Standards in Stroke Recovery Research: The Stroke Recovery and Rehabilitation Roundtable Taskforce. Neurorehabil Neural Repair 2017; 31: 793-799.

31. Monticone M, Ambrosini E, Ferrante S, et al. 'Regent Suit' training improves recovery of motor and daily living activities in subjects with subacute stroke: a randomized controlled trial. Clin Rehabil 2013; 27: 792-802.

32. Peri E, Ambrosini E, Pedrocchi A, et al. Can FES-Augmented Active Cycling Training Improve Locomotion in Post-Acute Elderly Stroke Patients? Eur J Transl Myol 2016; 26: 6063 .

33. Ferrante S, Ambrosini E, Ravelli P, et al. A biofeedback cycling training to improve locomotion: a case series study based on gait pattern classification of 153 chronic stroke patients. J Neuroeng Rehabil 2011; 8: 47.

34. Fulk GD, Echternach JL, Nof L, et al. Clinometric properties of the six-minute walk test in individuals undergoing rehabilitation poststroke. Physiother Theory Pract 2008; 24: 195-204.

35. Teasell R, McRae M, Foley N, et al. The incidence and consequences of falls in stroke patients during inpatient rehabilitation: factors associated with high risk. Arch Phys Med Rehabil 2002; 83: 329-33.

36. Cameron D, Bohannon RW. Criterion validity of lower extremity Motricity Index scores. Clin Rehabil 2000; 14: 208-11.

37. Collin C, Wade D. Assessing motor impairment after stroke: a pilot reliability study. $J$ Neurol Neurosurg Psychiatry 1990; 53: 576-9.

38. Dewan N, MacDermid JC. Fall Efficacy Scale - International (FES-I). J Physiother 
2014; 60: 60 .

39. Kamper SJ, Ostelo RWJG, Knol DL, et al. Global Perceived Effect scales provided reliable assessments of health transition in people with musculoskeletal disorders, but ratings are strongly influenced by current status. J Clin Epidemiol 2010; 63: 760766.e1.

40. Tilson JK, Sullivan KJ, Cen SY, et al. Meaningful gait speed improvement during the first 60 days poststroke: minimal clinically important difference. Phys Ther 2010; 90: 196-208.

41. Fulk GD, He Y. Minimal Clinically Important Difference of the 6-Minute Walk Test in People With Stroke. J Neurol Phys Ther 2018; 42: 235-240.

42. Perry J, Garrett M, Gronley JK, et al. Classification of walking handicap in the stroke population. Stroke 1995; 26: 982-9.

43. French B, Thomas LH, Coupe J, et al. Repetitive task training for improving functional ability after stroke. Cochrane Database Syst Rev 2016; 2016: 102-104.

44. Ambrosini E, Ferrante S, Schauer T, et al. A myocontrolled neuroprosthesis integrated with a passive exoskeleton to support upper limb activities. J Electromyogr Kinesiol 2014; $24: 307-317$.

45. Gandolla M, Ward NS, Molteni F, et al. The Neural Correlates of Long-Term Carryover following Functional Electrical Stimulation for Stroke. Neural Plast; 2016.

46. Barsi GI, Popovic DB, Tarkka IM, et al. Cortical excitability changes following grasping exercise augmented with electrical stimulation. Exp Brain Res 2008; 191: 5766.

47. Renner CI, Outermans J, Ludwig R, et al. Group therapy task training versus individual task training during inpatient stroke rehabilitation: a randomised controlled trial. Clin Rehabil 2016; 30: 637-648. 
48. Morone G, Bragoni M, Iosa M, et al. Who May Benefit From Robotic-Assisted Gait Training? Neurorehabil Neural Repair 2011; 25: 636-644.

49. Morone G, Iosa M, Bragoni M, et al. Who may have durable benefit from robotic gait training?: a 2-year follow-up randomized controlled trial in patients with subacute stroke. Stroke 2012; 43: 1140-2.

50. Zucchella C, Consilvio M, Iacoviello L, et al. Rehabilitation in oldest-old stroke patients: a comparison within over 65 population. Eur J Phys Rehabil Med 2019; 55: $148-155$. 


\section{NOTES}

\section{Conflicts of interest}

None.

\section{Funding}

The study was funded by the Italian Ministry of Healthy (Grant number: GR-2010-2312228).

Authors' contributions

- Emilia Ambrosini was responsible for conceiving and designing the study, integrating the setup, collecting, analysing and interpreting the data, drafting and critically revising the article, and approving the final version of the article.

- Elisabetta Peri was responsible for collecting, analysing and interpreting the data, critically revising the article, and approving the final version of the article.

- Claudia Nava was responsible for collecting the data, critically revising the article, and approving the final version of the article.

- Luca Longoni was responsible for collecting the data, critically revising the article, and approving the final version of the article.

- Marco Monticone was responsible for conceiving and designing the study, interpreting the data, critically revising the article, and approving the final version of the article.

- Alessandra Pedrocchi was responsible for conceiving and designing the study, critically revising the article, and approving the final version of the article.

- Giorgio Ferriero was responsible for interpreting the data, critically revising the article, and approving the final version of the article.

- Simona Ferrante was responsible for conceiving and designing the study, integrating the setup, analysing and interpreting the data, drafting and critically revising the article, and approving the final version of the article. 


\section{Acknowledgments}

The Authors would like to acknowledge the patients for participating in the study and Axelgaard Manufacturing Co., Ltd. for donating the stimulation electrodes. 


\section{TABLES}

Table I.- Demographic characteristics $(\mathrm{N}=68)$.

\begin{tabular}{|c|c|c|c|}
\hline & $\begin{array}{c}\text { Experimental } \\
\text { Group }\end{array}$ & $\begin{array}{l}\text { Control } \\
\text { Group }\end{array}$ & p-value \\
\hline $\mathbf{A g e}^{\mathbf{a}}$, years & $73.7(11.7)$ & $74.9(12.8)$ & $0.694^{\mathrm{b}}$ \\
\hline \multicolumn{4}{|l|}{ Distribution of age } \\
\hline$<65$ years & $8(23.5 \%)$ & $6(17.5 \%)$ & \\
\hline $65 \leq$ years $<75$ & $8(23.5 \%)$ & $5(15 \%)$ & \\
\hline $75 \leq$ years $<85$ & $13(38 \%)$ & $15(44 \%)$ & \\
\hline$\geq 85$ years & $5(15 \%)$ & $8(23.5 \%)$ & \\
\hline Time since disease onset ${ }^{\mathrm{a}}$, days & $13.9(5.0)$ & $18.0(14.3)$ & $0.129^{\mathrm{b}}$ \\
\hline Gender, male / female & $21 / 13$ & $17 / 17$ & $0.329^{c}$ \\
\hline Etiology, ischemic / hemorrhagic / hematoma & $29 / 4 / 1$ & $26 / 5 / 3$ & $0.529^{c}$ \\
\hline Hemiparetic side, right / left & $21 / 13$ & $15 / 19$ & $0.145^{\mathrm{c}}$ \\
\hline \multicolumn{4}{|l|}{ Comorbidity (principal) } \\
\hline Cardiac diseases & 11 & 17 & \\
\hline Diabetes & 7 & 1 & \\
\hline Respiratory diseases & 2 & 2 & \\
\hline Gastroenteric diseases & 4 & 1 & \\
\hline Endocrine diseases & 3 & 0 & \\
\hline Depression & 3 & 1 & \\
\hline Other & 3 & 7 & \\
\hline Mini-Mental State Examination ${ }^{a}[0-30]$ & $26(4)$ & $26(3)$ & $0.519^{\mathrm{b}}$ \\
\hline Not able / able to walk at TO & $5 / 29$ & $10 / 24$ & $0.144^{\mathrm{c}}$ \\
\hline Gait speed $^{\mathrm{a}}[\mathrm{cm} / \mathrm{s}]$ & $65.2(27.9)$ & $57.7(18.7)$ & $0.251^{\mathrm{b}}$ \\
\hline \multicolumn{4}{|l|}{${ }^{\mathrm{a}}$ Mean (standard deviation). } \\
\hline${ }^{\mathrm{b}} \mathrm{T}$-test for independent samples. & & & \\
\hline test. & & & \\
\hline
\end{tabular}



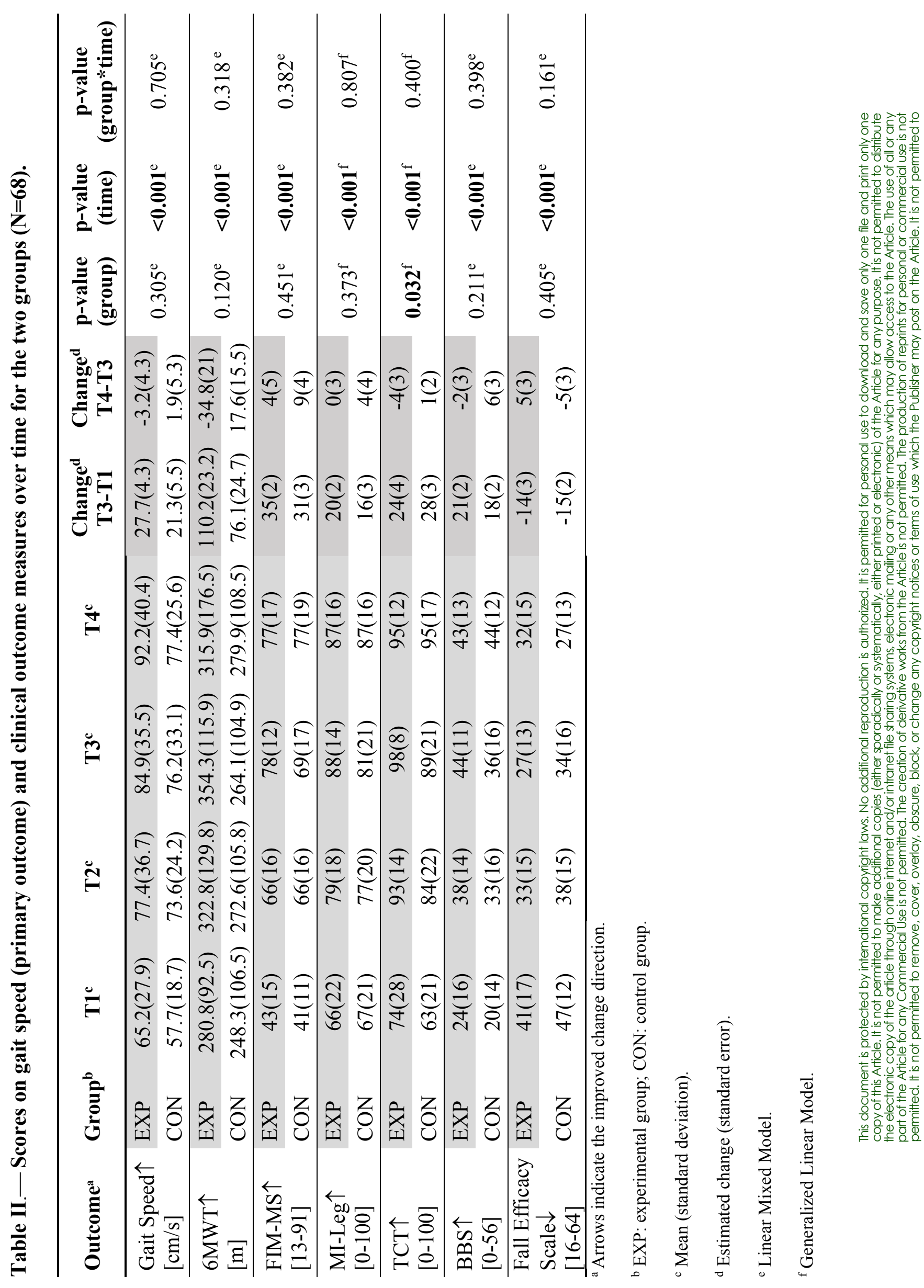

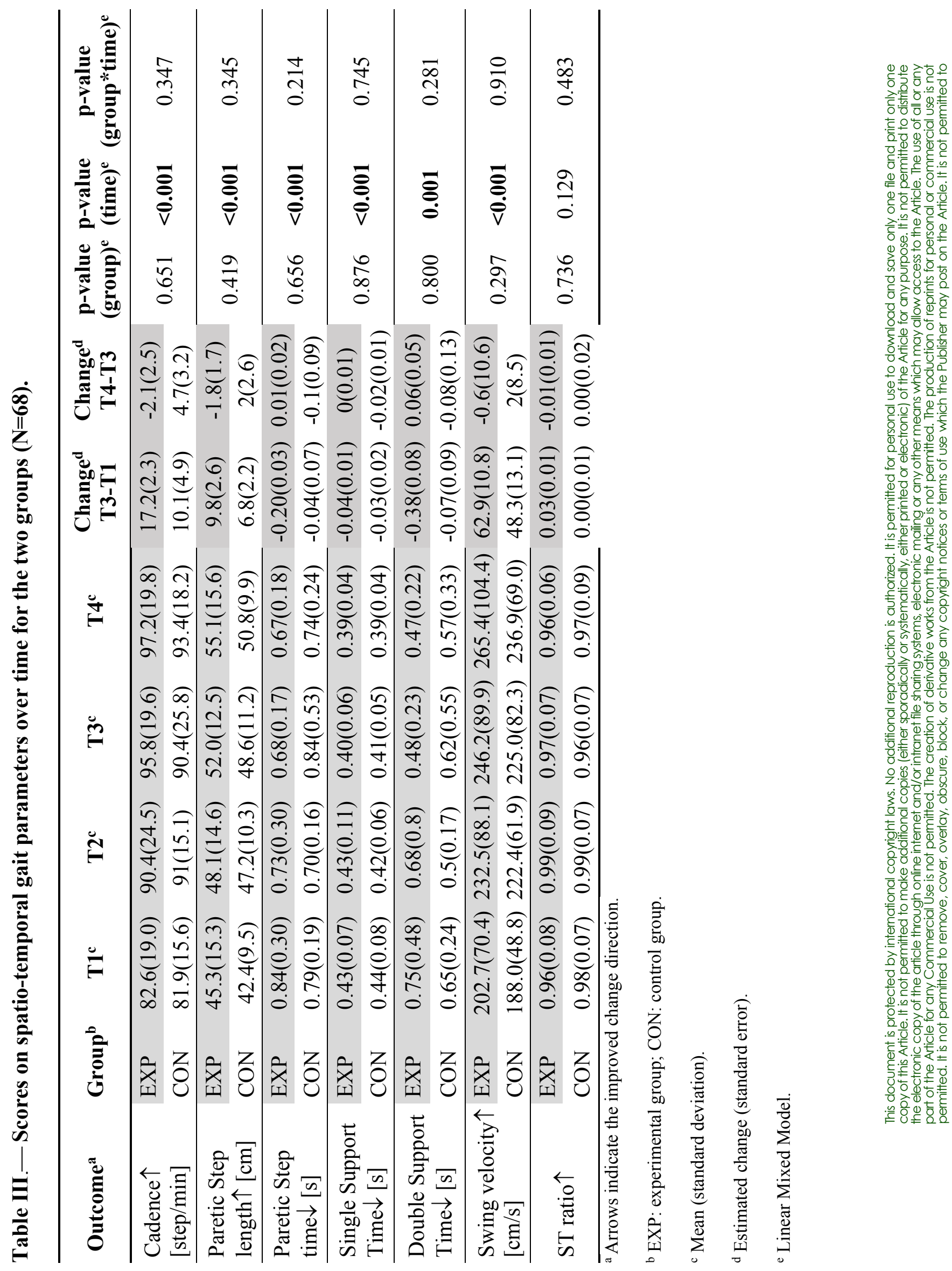


\section{TITLES OF FIGURE}

Figure 1.- Schematic of the interventions. The left panel shows the setup used for FEScycling training, the biomimetic stimulation strategy in terms of crank angular ranges of the right leg (Q: quadriceps; $\mathrm{H}$ : hamstrings; T: tibialis anterior; $\mathrm{G}$ : gastrocnemius lateralis) and the visual feedback shown to the subject. The right panel displays the setup and the visual feedback of the balance exercises: \#1: Upright posture; \#2: Rhythmic medio-lateral weight shifting; \#3: Rhythmic antero-posterior weight shifting; \#4: Reaching of pre-defined radial targets from central position; \#5: Right weight shift \& Left step forward; \#6: Left weight shift \& Right step forward. The lower arrow indicates the training and assessment timeline.

\section{Figure 2.- Participant CONSORT flow chart.}

Figure 3.- Percentage of participants who improved locomotor ability at T3. Panels (AC) show the percentage of subjects who clinically improved their gait speed; panels (D-F) show the percentage of subjects who clinically improved their walking endurance (6MWT). Panels (A) and (D) consider all patients who completed the T3 assessment. Panels (B) and (E) consider the sub-group of older subjects (age $\geq 75 y$ ears). Panels $(\mathrm{C})$ and $(\mathrm{F})$ take into account the subgroup of more impaired subjects at baseline (unable to walk or gait speed $<40 \mathrm{~cm} / \mathrm{s}$ ). EXP: experimental group; CON: control group. 


\section{CONTROL GROUP}

90-min of standard physical therapy

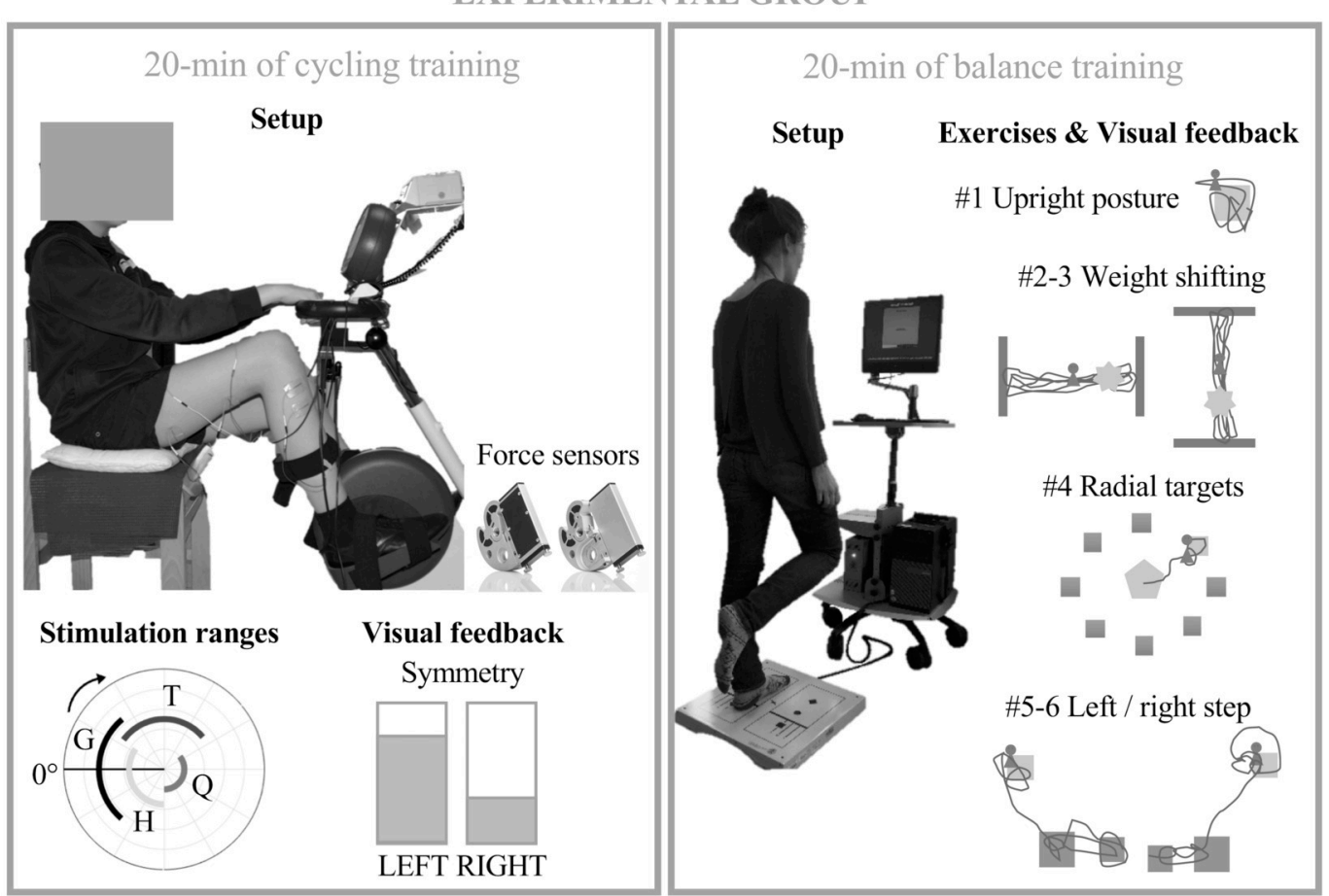

70-min of standard physical therapy

Weeks

\begin{tabular}{|c|c|c|c|}
\hline 0 & 3 & 6 & 30 \\
\hline T1 & $\mathbf{T 2}$ & T3 & T4 \\
\hline
\end{tabular}




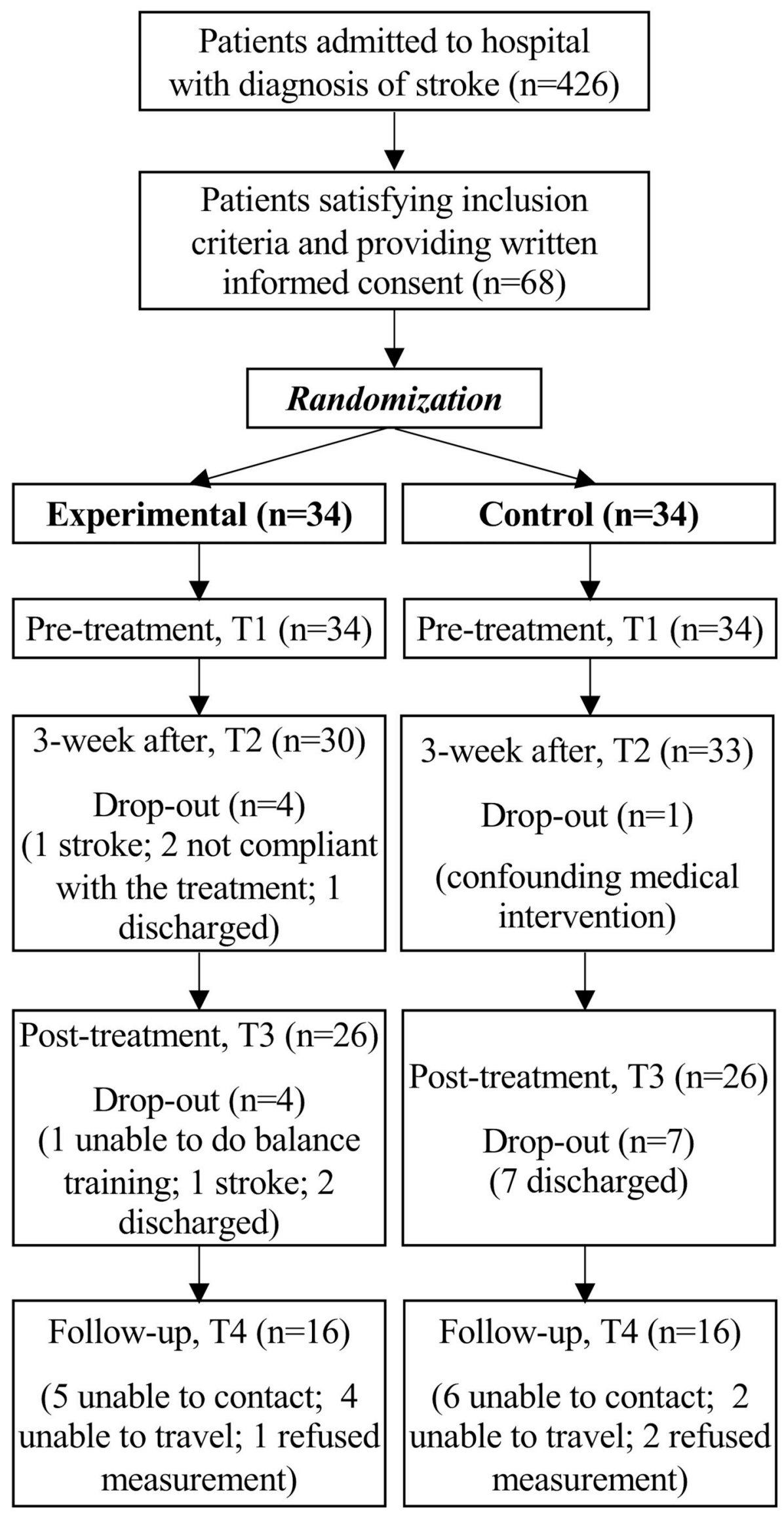


All subjects $(\mathrm{N}=52)$
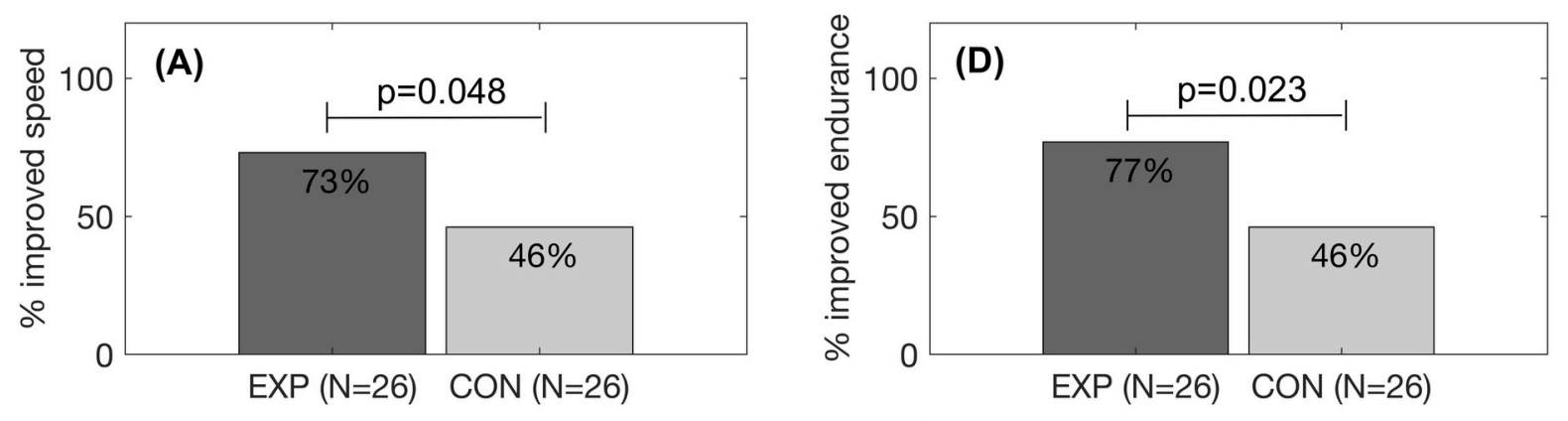

Older subjects $(\mathrm{N}=\mathbf{3 1})$
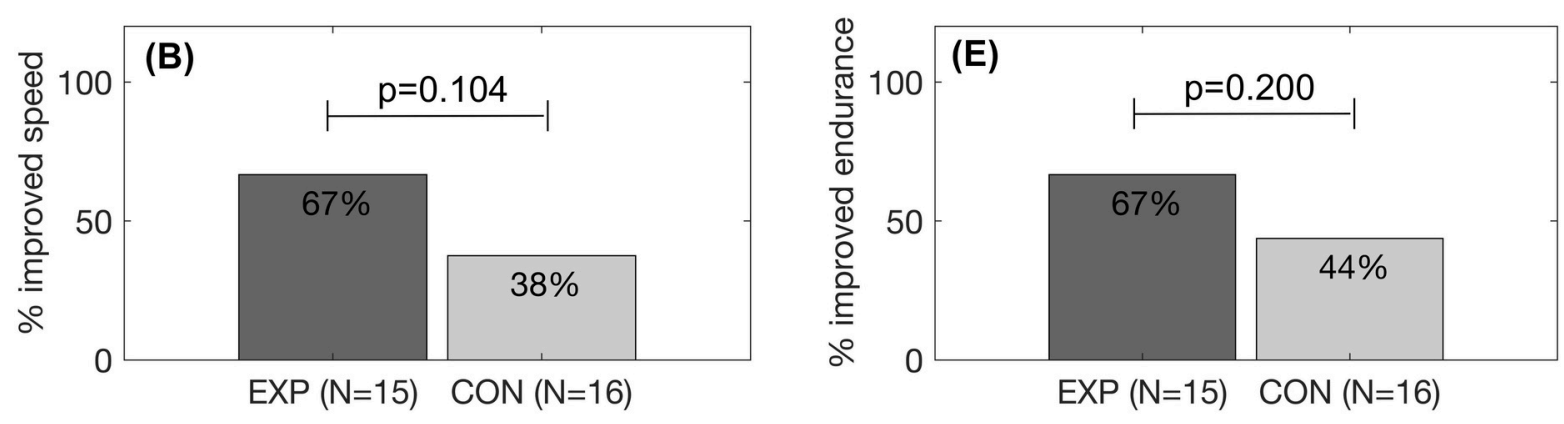

More impaired subjects ( $\mathrm{N}=22$ )
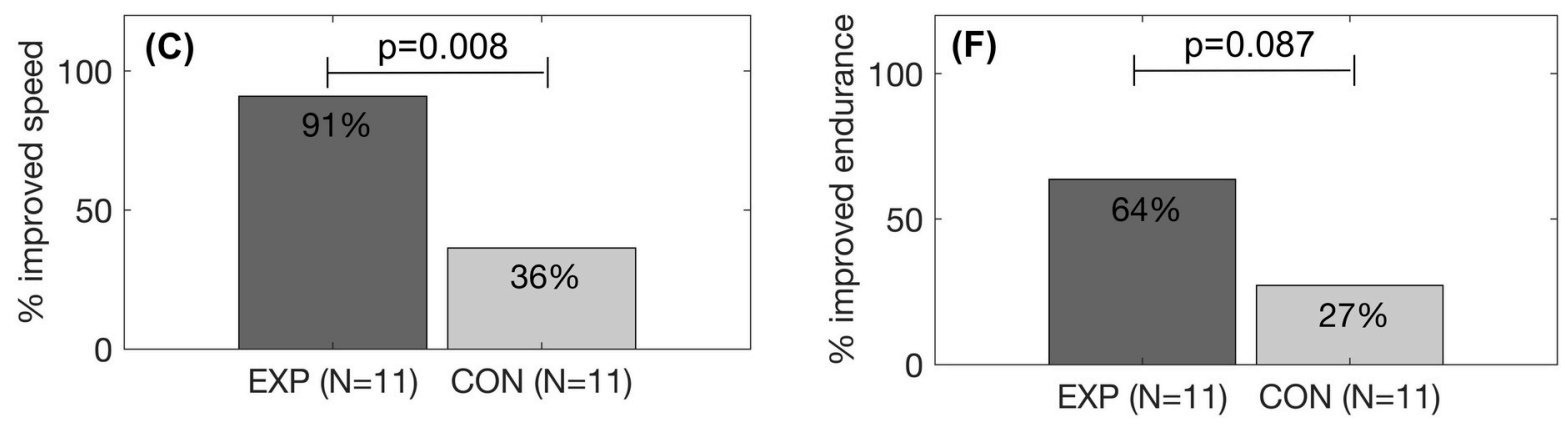